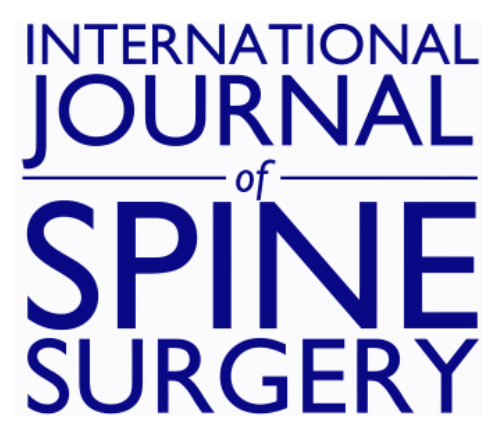

\title{
Multimodal Analgesic Management for Cervical Spine Surgery in the Ambulatory Setting
}

Nathaniel W. Jenkins, James M. Parrish, Michael T. Nolte, Caroline N. Jadczak, Shruthi Mohan, Cara E. Geoghegan, Nadia M. Hrynewycz, Jeffrey Podnar, Asokumar Buvanendran and Kern Singh

Int J Spine Surg 2021, 15 (2) 219-227

doi: https://doi.org/10.14444/8030

http://ijssurgery.com/content/15/2/219

This information is current as of April 26, 2023.

Email Alerts Receive free email-alerts when new articles cite this article. Sign up at: http://ijssurgery.com/alerts 


\title{
Multimodal Analgesic Management for Cervical Spine Surgery in the Ambulatory Setting
}

\author{
NATHANIEL W. JENKINS, MS, ${ }^{1}$ JAMES M. PARRISH, MPH, ${ }^{1}$ MICHAEL T. NOLTE, MD, ${ }^{1}$ CAROLINE N. \\ JADCZAK, BS, ${ }^{1}$ SHRUTHI MOHAN, BS, ${ }^{1}$ CARA E. GEOGHEGAN, BS,${ }^{1}$ NADIA M. HRYNEWYCZ, BS, ${ }^{1}$ \\ JEFFREY PODNAR, MD, ${ }^{2}$ ASOKUMAR BUVANENDRAN, MD, ${ }^{3}$ KERN SINGH, MD ${ }^{1}$ \\ Departments of ${ }^{1}$ Orthopaedic Surgery and ${ }^{3}$ Anesthesiology, Rush University Medical Center, Chicago, Illinois, ${ }^{2}$ Department of Anesthesiology, Midwest \\ Anesthesia Partners LLC, Park Ridge, Illinois
}

\begin{abstract}
Background: Patient selection and analgesic techniques, such as the multimodal analgesic (MMA) protocol, aid in ambulatory surgical center (ASC) cervical spine surgery. The purpose of this case series is to characterize patients undergoing anterior cervical discectomy and fusion (ACDF) and total cervical disc replacement (CDR) in an ASC with an enhanced MMA protocol.

Methods: A prospectively maintained registry was retrospectively reviewed for cervical surgeries between May 2013 and August 2019. Inclusion criteria included ASC patients who underwent single-level or multilevel CDR or ACDF using an MMA protocol. Baseline, intraoperative, and postoperative characteristics were recorded, including length of stay, visual analog scale pain scores, neck disability index, complications, and narcotics administered.

Results: A total of 178 patients met inclusion criteria with 125 single-level, 52 two-level, and 1 three-level procedure. Of those patients, 127 underwent ACDF and 51 underwent CDR. The longest procedure was 95 minutes and the mean length of stay was 6.1 hours, with 2 patients requiring hospital admission. All other patients were discharged within 10 hours. One of the admitted patients experienced a postoperative seizure that was later determined to be secondary to drug use and serotonin syndrome. The second patient developed an anterior cervical hematoma 5 hours postoperatively, which was immediately evacuated. The patient was admitted for observation and discharged the next day.

Conclusion: In our study, patients experienced considerable improvement in disability scores, with a low likelihood of postoperative complications. A safe and effective MMA protocol may help facilitate anterior cervical surgery in the outpatient setting.
\end{abstract}

Level of Evidence: 3.

Clinical Relevance: Transitioning anterior cervical discectomy and fusions to the ASC requires an appropriate MMA protocol. Our findings reveal that an enhanced MMA protocol will help improve disability scores while keeping the likelihood of postoperative complications low. This supports the ASC setting for cervical spine procedures in appropriate patient populations.

Cervical Spine

Keywords: cervical spine surgery, anterior cervical discectomy and fusion, cervical disc replacement, multimodal analgesia

\section{INTRODUCTION}

Anterior cervical spine surgery, namely anterior cervical discectomy and fusion (ACDF) and total cervical disc replacement (CDR), comprises wellstudied treatments for degenerative conditions of the cervical spine that have been observed to facilitate excellent longitudinal clinical outcomes. Furthermore, improvements in minimally invasive surgery, along with advances in anesthesia and analgesic techniques, have enabled these surgeries to take place in the outpatient setting and ambulatory surgery centers (ASC) more than ever before. One of the key barriers to widespread adoption, however, is ensuring safe and sufficient pain control. Appropriate management of pain has been associated with both increased patient satisfaction and lower complication rates. ${ }^{1,2}$ Furthermore, when compared with the inpatient hospital setting, ambulatory cervical spine surgery has been associated with superior short-term outcomes, lower complication rates, and lower direct costs related to the procedure. $^{3-6}$ Although anesthesia-related factors such as preoperative diet, patient optimization, and avoid- 
Table 1. Multimodal analgesic regimen for outpatient spine surgery.

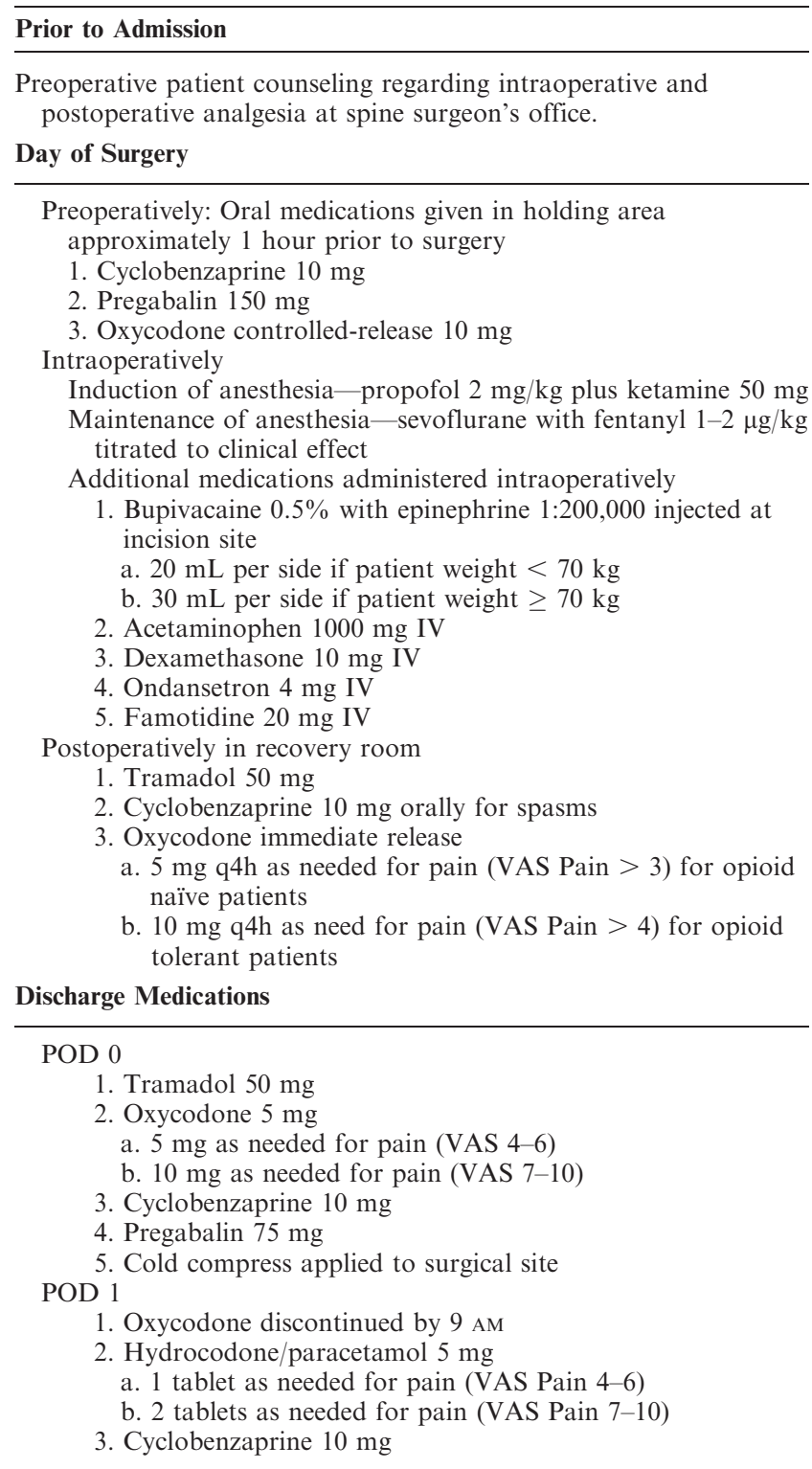

Abbreviations: POD, postoperative day; $\mathrm{q} 4 \mathrm{~h}$, every 4 hours; VAS, visual analog scale for pain (where $0=$ no pain and $10=$ worst possible pain).

ance of extended preoperative fasting have all increased the ability to perform ambulatory surgery, multimodal analgesia (MMA) is the key differentiating factor associated with more rapid recovery, decreased opioid use, a lower rate of complications, and increased patient satisfaction. ${ }^{3,7}$

Prior research efforts regarding cervical spine surgery in the outpatient setting have focused on the characterization and avoidance of complications, patient selection criteria, and inpatient admission rates following surgery. ${ }^{5,8-13}$ Studies examining the role of an MMA protocol have been limited. Although some efforts have analyzed the effectiveness of MMA protocols for cervical spine surgery in the inpatient setting, the outpatient and ASC settings pose a number of unique challenges that warrant separate investigation. In this study, we highlight a detailed MMA protocol in the ASC setting and report findings from our initial clinical experience. We believe that doing so may help guide surgical teams aiming to grow and streamline their anterior cervical spine surgeries in the ASC setting.

\section{METHODS}

\section{Patient Population}

Following institutional review board approval (ORA No. 14051301), we performed a retrospective review of consecutive patients undergoing anterior cervical spine surgery, either ACDF or CDR, from a prospectively maintained surgical registry. All surgeries were performed between May 2013 and November 2018 by a single senior surgeon at our institution. Patients included in the study were treated for degenerative spinal pathologies. Empiric medical treatment for these conditions was attempted without symptom relief for all patients prior to operative management. Nonsurgical therapy included the use of anti-inflammatory medications, corticosteroid injections, local anesthetics, and physical therapy. Prior to undergoing cervical spine surgery, each patient was assessed and cleared for surgery by an anesthesiologist and primary care physician.

All patients underwent either a primary or revision single-level or multilevel ACDF or CDR using a standard anterior approach to the cervical spine. Prophylaxis for deep venous thrombosis was achieved through the use of ambulation, and no chemical prophylaxis was used. All patients received a unique MMA protocol specifically designed for the ambulatory setting (Table 1). The ASC did not allow for observation of patients for periods of time greater than 23 hours. All patients who underwent cervical surgery were required to receive a same-day discharge. Per state regulations, the ASC was located within 30 minutes of a hospital with inpatient and intensive care unit capabilities. In the event of an emergency that cannot be adequately treated at the ASC, patients would be transferred to this hospital via ambulance.

\section{Data Collection}

Patient demographic factors recorded included age, sex, body mass index (BMI), smoking status, 
Charlson Comorbidity Index (CCI), American Society of Anesthesiologists (ASA) score, and preoperative diagnosis. These diagnoses included cervical spinal stenosis, degenerative disc disease, degenerative spondylolisthesis, foraminal stenosis, herniated nucleus pulposus, myelomalacia, myelopathy, myeloradiculopathy, and radiculopathy. Preoperative medical conditions that were recorded at the time of the medical clearance appointment, including arthritis, asthma, cancer, diabetes, hyperlipidemia, hypertension, liver disease, and peripheral vascular disease, were also recorded.

Perioperative characteristics that were recorded included operative location, operative time, estimated blood loss, and ambulatory center length of stay. Patient-reported outcomes including the visual analog scale (VAS) pain score and neck disability index (NDI) score were recorded. Narcotic consumption prior to discharge was calculated following a standard conversion into units of oral morphine equivalents (OME). Postoperative complications were recorded, including acute renal failure, airway obstruction, altered mental status, atelectasis, atrial fibrillation, atrial flutter, aspiration, epidural hematoma, ileus, nausea and vomiting, postoperative anemia requiring transfusion, pulmonary embolism, pneumothorax, seizure of unknown origin, urinary retention, urinary tract infection, and venous thromboembolism.

\section{Surgical Technique}

Patients were intubated and placed on the operating table in the supine position. The surgical level was confirmed and localized with intraoperative radiographic fluoroscopy. Injection of local anesthetic, bupivacaine $0.5 \%$ with epinephrine 1:200 000, was made to each surgical site prior to incision. A 2- to $3-\mathrm{cm}$ transverse incision, medial to the sternocleidomastoid muscle, was made through the skin and subcutaneous fat. The Smith-Robinson approach was used, and the platysma muscle was transversely dissected with an incision that aligned with that of the skin. Next, the sternocleidomastoid muscle and carotid sheath were laterally retracted, and the esophagus, trachea, and thyroid were medially retracted. Further blunt dissection was conducted down to the vertebral body and the pertinent disc space was identified.

Fluoroscopic radiographs were again used to confirm the surgical level. The annulus was then incised. Curved and straight curettes were used to remove the disc material and end-plate cartilage. Resection of the posterior longitudinal ligament was then accomplished with a Kerrison rongeur. Adequate preparation of the disc space was ensured. For patients undergoing ACDF, local autograft, allograft, or bone graft substitute was used to fill an appropriately sized interbody cage, and the cage was placed into the disc space. To help prevent interbody movement and subsidence, supplemental plate fixation was used. For patients undergoing CDR, following disc space preparation various size trials were used and checked under fluoroscopic guidance. Once the ideal size was agreed upon, the CDR implant was placed into the disc space. Once instrumentation was finished, the wound was thoroughly irrigated and evaluated for hemostasis.

\section{RESULTS \\ Demographic Characteristics}

A total of 178 patients met inclusion criteria. Of these, 125 patients underwent a 1-level procedure, 52 patients underwent a 2-level procedure, and 1 patient underwent a 3-level procedure $(71.3 \%$ underwent ACDF, 28.7\% underwent CDR; Table 2). The overall cohort consisted of $63.5 \%$ men with a mean age of $46.7 \pm 9.1$ years. Mean BMI was 28.6 $\pm 4.4 \mathrm{~kg} / \mathrm{m}^{2}$, and 22 patients reported tobacco use at their preoperative appointment. The mean CCI was $0.46 \pm 0.8$, with the majority of patients $(50.5 \%)$ having an ASA score of 2. Preoperative chronic medical conditions and comorbidities were as follows: hypertension (29), asthma (15), hyperlipidemia (3), cancer (2), uncomplicated diabetes mellitus (8), liver disease (1), and peripheral vascular disease (1). Of note, there were no patients in our cohort with a recorded medical history of myocardial infarction, chronic lung disease, renal failure, or gastrointestinal bleeding.

\section{Perioperative and Postoperative Characteristics}

A total of 127 patients underwent an ACDF, of which $92.7 \%$ were primary procedures (Tables 3 and 4). A total of 51 patients underwent a CDR, of which $90.2 \%$ were primary procedures. The most common preoperative diagnosis was myeloradiculopathy for both patients undergoing ACDF $(59.8 \%)$ and those undergoing CDR $(24.1 \%)$.

The most common operative level was C6-C7 (30.9\%; Table 5). The longest surgical case was 95 
Table 2. Patient demographics and baseline characteristics.

\begin{tabular}{|c|c|c|c|c|}
\hline & Total $(N=178)^{a}$ & 1 Level $(n=125)$ & 2 Level $(n=52)$ & $\geq 3$ Level $(\mathbf{n}=\mathbf{1})$ \\
\hline Age, mean $\pm \mathrm{SD}, \mathrm{y}$ & $46.7 \pm 9.1$ & $45 \pm 8.9$ & $52.2 \pm 8.8$ & 44.0 \\
\hline \multicolumn{5}{|l|}{ Gender, \% (n) } \\
\hline Female & $36.5(65)$ & $36(45)$ & $38.5(20)$ & $0.0(0)$ \\
\hline Male & $63.5(113)$ & $64(80)$ & $61.5(32)$ & $100.0(1)$ \\
\hline Body mass index (mean $\left.\pm \mathrm{SD}, \mathrm{kg} / \mathrm{m}^{2}\right)$ & $28.6 \pm 4.4$ & $29.0 \pm 4.5$ & $27.6 \pm 4.2$ & 29.8 \\
\hline \multicolumn{5}{|l|}{ Smoking status, \% (n) } \\
\hline Nonsmoker & $87.4(153)$ & $86.2(106)$ & $90.2(46)$ & $100.0(1)$ \\
\hline Smoker & $12.6(22)$ & $13.8(17)$ & $9.8(5)$ & $0.0(0)$ \\
\hline Charlson Comorbidity Index, mean $\pm \mathrm{SD}$ & $0.46 \pm 0.8$ & $0.47 \pm 0.8$ & $0.44 \pm 0.6$ & $0.0 \pm 0.0$ \\
\hline \multicolumn{5}{|l|}{ ASA score, $\%(n)$} \\
\hline 1 & $45.0(49)$ & $43.4(32)$ & $47.1(16)$ & $100.0(1)$ \\
\hline 2 & $50.5(55)$ & $54.1(40)$ & $44.1(15)$ & $0.0(0)$ \\
\hline$\geq 3$ & $4.6(5)$ & $2.7(2)$ & $8.8(3)$ & $0.0(0)$ \\
\hline \multicolumn{5}{|l|}{ Preoperative diagnoses, $\%(n)^{b}$} \\
\hline Hypertension & $16.6(29)$ & $14.6(18)$ & $21.6(11)$ & $0.0(0)$ \\
\hline Asthma & $8.4(15)$ & $8.8(11)$ & $7.7(4)$ & $0.0(0)$ \\
\hline Arthritis & $7.4(13)$ & $8.1(10)$ & $5.9(3)$ & $0.0(0)$ \\
\hline Hyperlipidemia & $1.7(3)$ & $0.8(1)$ & $3.9(2)$ & $0.0(0)$ \\
\hline Cancer & $1.1(2)$ & $1.6(2)$ & $0.0(0)$ & $0.0(0)$ \\
\hline Uncomplicated diabetes mellitus & $4.6(8)$ & $3.3(4)$ & $7.8(4)$ & $0.0(0)$ \\
\hline Liver disease & $0.6(1)$ & $0.8(1)$ & $0.0(0)$ & $0.0(0)$ \\
\hline Peripheral vascular disease & $0.6(1)$ & $0.8(1)$ & $0.0(0)$ & $0.0(0)$ \\
\hline
\end{tabular}

Abbreviation: ASA, American Society of Anesthesiologists.

${ }^{a}$ Percentages were based on total $\mathrm{n}$ of patients without missing data; those that had $\mathrm{n}<178$ include hypertension, arthritis, cancer, uncomplicated diabetes mellitus, liver disease $(n=175)$, peripheral vascular disease $(n=174)$.

${ }^{\mathrm{b}}$ There were no patients in our study with a recorded medical history of myocardial infarction, renal failure, chronic lung disease, or gastrointestinal bleeding.

minutes with 1 outlier for length of stay at 23 hours (first cervical procedure performed at the ASC). The mean length of stay was $6.1 \pm 2.5$ hours, and 2 patients required admission at a local hospital. All patients, aside from the first, were discharged within 10 hours of the procedure end. The mean postoperative VAS pain score prior to discharge was $5.1 \pm$ 2.5. The mean narcotics consumed following surgery and prior to discharge was $31.4 \pm 17.6$ OME. The cohort reported considerable improvement in

Table 3. Preoperative spinal diagnoses $(\mathrm{N}=178)$.

\begin{tabular}{|c|c|c|c|c|}
\hline & \multicolumn{4}{|c|}{ Cervical Procedures, $N=178^{a}$} \\
\hline & \multicolumn{2}{|c|}{$\mathrm{ACDF}, \mathbf{n}=127$} & \multicolumn{2}{|c|}{ CDR, $\mathrm{n}=\mathbf{5 1}$} \\
\hline & $\begin{array}{l}\text { Primary, } \\
\% \text { (n) }\end{array}$ & $\begin{array}{l}\text { Revision, } \\
\% \text { (n) }\end{array}$ & $\begin{array}{c}\text { Primary, } \\
\% \text { (n) }\end{array}$ & $\begin{array}{l}\text { Revision, } \\
\% \text { (n) }\end{array}$ \\
\hline Herniated nucleus pulposus & $49.4(86)$ & $1.7(3)$ & $16.7(29)$ & $0.6(1)$ \\
\hline Degenerative disc disease & $3.4(6)$ & $2.3(4)$ & $2.9(5)$ & $0.0(0)$ \\
\hline Cervical spinal stenosis & $35.1(61)$ & $4.6(8)$ & $12.1(21)$ & $1.7(3)$ \\
\hline Myelomalacia & $0.6(1)$ & $0.0(0)$ & $0.0(0)$ & $0.0(0)$ \\
\hline Degenerative Spondylolisthesis & $0.0(0)$ & $0.6(1)$ & $0.0(0)$ & $0.6(1)$ \\
\hline Foraminal stenosis & $6.3(11)$ & $2.3(4)$ & $1.1(2)$ & $0.0(0)$ \\
\hline Radiculopathy & $5.7(10)$ & $0.6(1)$ & 1. (2) & $0.6(1)$ \\
\hline Myelopathy & $0.0(0)$ & $0.0(0)$ & $0.6(1)$ & $0.0(0)$ \\
\hline Myeloradiculopathy & $59.8(104)$ & $2.9(5)$ & $24.1(42)$ & $1.7(3)$ \\
\hline
\end{tabular}

NDI during the postoperative period at 6 weeks $(32.1 \pm 19.3), 12$ weeks $(29.5 \pm 18.9), 6$ months $(28.2 \pm 19.4)$, and 1 year $(26.4 \pm 20.4)$.

A total of 6 complications were observed in patients during the immediate postoperative period in the ASC (Table 6). Postoperative nausea and vomiting constituted 4 of the complications. All 4 of these patients were discharged in less than 23 hours and did not require admission. Two patients required admission to the hospital following surgery. One of these patients used illicit drugs prior to surgery, a practice unbeknown to our team or the anesthesia providers, and experienced a postoperative seizure of unknown origin. The patient was admitted to a local academic hospital, and the seizures were subsequently determined to be secondary to serotonin syndrome. The second admitted patient developed an anterior cervical hematoma that was noted 5 hours postoperatively during the

Table 4. Total procedures: primary and revisions $(N=178){ }^{a}$

\begin{tabular}{lcc}
\hline & Primary, \% (n) & Revision, \% (n) \\
\hline ACDF $(\mathrm{n}=127)$ & $66.1(115)$ & $5.2(9)$ \\
CDR $(\mathrm{n}=51)$ & $26.4(46)$ & $2.3(4)$ \\
\hline
\end{tabular}

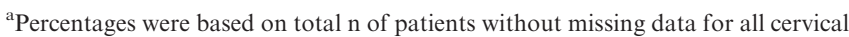
procedures; revision data was limited $(\mathrm{n}=174)$.

${ }^{b}$ Revisions were considered in any case of revision (eg, primary fusion with a revision decompression, a complete revision procedure, reoperation, or additional procedure). 
Table 5. Perioperative characteristics.

\begin{tabular}{|c|c|c|c|c|}
\hline & Total $(\mathrm{n}=178)^{\mathrm{a}}$ & 1 Level $(n=125)$ & 2 Level $(n=52)$ & $\geq 3$ Level $(n=1)$ \\
\hline \multicolumn{5}{|l|}{ Operative location, \% (n) } \\
\hline $\mathrm{C} 3-\mathrm{C} 4$ & $2.8(5)$ & $4(5)$ & $0.0(0)$ & $0.0(0)$ \\
\hline $\mathrm{C} 4-\mathrm{C} 5$ & $5.1(9)$ & $7.2(9)$ & $0.0(0)$ & $0.0(0)$ \\
\hline $\mathrm{C} 4-\mathrm{C} 6$ & $7.3(13)$ & $0.0(0)$ & $25.0(13)$ & $0.0(0)$ \\
\hline $\mathrm{C} 4-\mathrm{C} 7$ & $0.6(1)$ & $0.0(0)$ & $0.0(0)$ & $100.0(1)$ \\
\hline C5-C6 & $29.8(53)$ & $42.4(53)$ & $0.0(0)$ & $0.0(0)$ \\
\hline $\mathrm{C} 5-\mathrm{C} 7$ & $22.5(40)$ & $1.6(2)$ & $73.1(38)$ & $0.0(0)$ \\
\hline C6-C7 & $30.9(55)$ & $44.0(55)$ & $0.0(0)$ & $0.0(0)$ \\
\hline C6-T1 & $0.6(1)$ & $0.0(0)$ & $1.9(1)$ & $0.0(0)$ \\
\hline C7-T1 & $0.6(1)$ & $0.9(1)$ & $0.0(0)$ & $0.0(0)$ \\
\hline Operative time $^{\mathrm{b}}$, mean $\pm \mathrm{SD}$, min & $63.2 \pm 56.8$ & $60.7 \pm 67.3$ & $69.1 \pm 7.8$ & 83.0 \\
\hline Estimated blood loss, mean $\pm \mathrm{SD}, \mathrm{mL}$ & $27.7 \pm 9.6$ & $27.2 \pm 7.3$ & $29.1 \pm 13.8$ & 25.0 \\
\hline Surgery center length of stay, mean $\pm \mathrm{SD}, \mathrm{h}$ & $6.1 \pm 2.5$ & $5.9 \pm 2.3$ & $6.3 \pm 2.9$ & 6.7 \\
\hline \multicolumn{5}{|l|}{ VAS pain scores, mean \pm SD } \\
\hline POD 0 & $5.1 \pm 2.5$ & $5.0 \pm 2.5$ & $5.4 \pm 1.9$ & 6.4 \\
\hline \multicolumn{5}{|l|}{ Narcotic consumption, mean $\pm \mathrm{SD}, \mathrm{OME}$} \\
\hline POD 0 & $31.4 \pm 17.6$ & $29.9 \pm 17.3$ & $34.3 \pm 17.9$ & 51 \\
\hline \multicolumn{5}{|l|}{$\mathrm{NDI}$, mean $\pm \mathrm{SD}$} \\
\hline Preoperative & $40.8 \pm 18.5$ & $40.5 \pm 17.6$ & $40.9 \pm 20.8$ & 50 \\
\hline $6 \mathrm{wk}$ & $32.1 \pm 19.3$ & $32.8 \pm 19.9$ & $30.7 \pm 18.0$ & 30 \\
\hline $12 \mathrm{wk}$ & $29.5 \pm 18.9$ & $30.1 \pm 19.3$ & $28.3 \pm 16.8$ & 32 \\
\hline $6 \mathrm{mo}$ & $28.2 \pm 19.4$ & $28.8 \pm 20.7$ & $26.7 \pm 15.0$ & 14 \\
\hline $1 \mathrm{y}$ & $26.4 \pm 20.4$ & $28.7 \pm 20.8$ & $19.8 \pm 18.7$ & - \\
\hline
\end{tabular}

Abbreviations: NDI, Neck Disability Index; OME, oral morphine equivalents; POD, postoperative day; VAS, visual analog scale.

${ }^{a}$ Percentages were based on total $\mathrm{n}$ of patients without missing data; those that had $\mathrm{n}<178$ include estimated blood loss $(\mathrm{n}=170)$, hospital length of stay $(\mathrm{n}=165)$, VAS postoperative day zero average $(\mathrm{n}=114)$, OME postoperative day zero average $(\mathrm{n}=114)$, preoperative NDI $(\mathrm{n}=153), 6$-wk NDI $(\mathrm{n}=132), 12$-wk NDI $(\mathrm{n}=112), 6$-mo NDI $(\mathrm{n}=88), 1-\mathrm{y}$ NDI $(\mathrm{n}=34)$

${ }^{\mathrm{b}}$ There were no patients in our study with a recorded medical history of myocardial infarction, renal failure, chronic lung disease, or gastrointestinal bleeding.

observational period. The hematoma caused shortness of breath concerning for airway obstruction and was immediately evacuated at the ASC. The patient was subsequently admitted to a local academic hospital for 23-hour observation. Both patients were uneventfully discharged the next day following surgery.

Table 6. Postoperative complications.

\begin{tabular}{|c|c|c|c|c|}
\hline Complications & $\begin{array}{c}\text { Total } \\
(\mathrm{N}=178)\end{array}$ & $\begin{array}{c}1 \text { Level } \\
(n=125)\end{array}$ & $\begin{array}{c}2 \text { Level } \\
(n=52)\end{array}$ & $\begin{array}{c}\geq 3 \text { Level } \\
(n=1)\end{array}$ \\
\hline & 0 & 0 & 0 & 0 \\
\hline Acute renal failure & 0 & 0 & 0 & 0 \\
\hline Airway obstruction $^{\mathrm{a}}$ & 1 & 0 & 1 & 0 \\
\hline Altered mental status & 0 & 0 & 0 & 0 \\
\hline Aspiration & 0 & 0 & 0 & 0 \\
\hline Epidural hematoma & 0 & 0 & 0 & 0 \\
\hline Ileus & 0 & 0 & 0 & 0 \\
\hline Nausea and vomiting $^{\mathrm{b}}$ & 4 & 3 & 1 & 0 \\
\hline Postoperative anemia & 0 & 0 & 0 & 0 \\
\hline Seizure of unknown origin ${ }^{c}$ & 1 & 1 & 0 & 0 \\
\hline Urinary retention & 0 & 0 & 0 & 0 \\
\hline Urinary tract infection & 0 & 0 & 0 & 0 \\
\hline Venous thromboembolism & 0 & 0 & 0 & 0 \\
\hline
\end{tabular}

${ }^{\text {a }}$ Single patient received immediate evacuation of cervical hematoma that developed at $5 \mathrm{~h}$ postoperatively, transferred to inpatient hospital for observation, and was uneventfully discharged following morning.

${ }^{\mathrm{b}}$ All patients discharged in less than $23 \mathrm{~h}$; no admissions or further hospitalizations required.

${ }^{\mathrm{c}}$ Single patient was emergently transferred with seizures postoperatively and diagnosed with serotonin surge secondary to illicit drug use prior to surgery.

\section{DISCUSSION}

\section{Patient Selection}

Successful cervical spine surgery in the ambulatory setting begins with appropriate patient selection. Careful screening is essential due to the diminished number of care providers and emergency services compared with the inpatient hospital setting. Exclusion criteria such as obesity, history of chronic obstructive pulmonary disease, hypertension, and stroke have been previously suggested as a general guideline for outpatient surgery, but more specific criteria is needed for cervical surgery. ${ }^{14}$ Unfortunately, there has been a paucity of literature guiding successful patient selection specifically for cervical spine surgery in the ambulatory surgical setting.

In light of this, we have proposed multifaceted recommendations for patient selection for cervical surgery in the ASC setting (Table 7). These are based not only on our clinical experience with 178 patients but also the available literature from the inpatient and nonambulatory setting. For example, Bovonratwet et $\mathrm{al}^{8}$ observed that patients with the following risk factors were more likely to experience postoperative hematoma requiring reoperation following ACDF: preoperative international normalized ratio $(\mathrm{INR})>1.2$ relative risk $[\mathrm{RR}]=2.85$, 
Table 7. Recommendations for patient selection for cervical surgery in ASC settings.

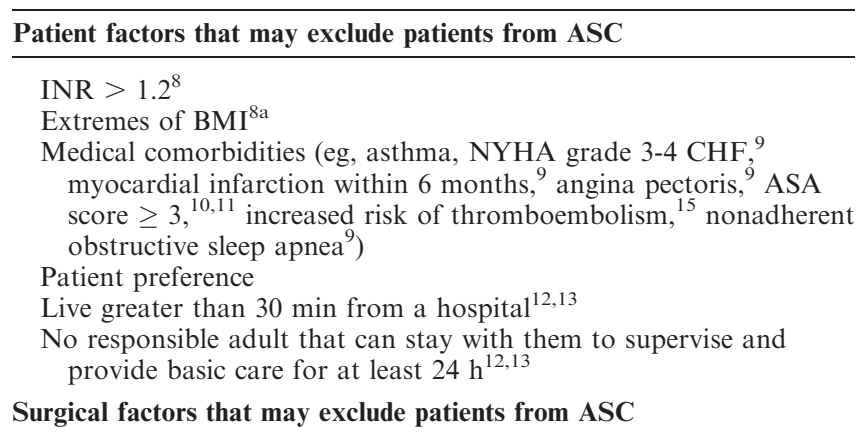

Operative time $(>5 \mathrm{~h})$

EBL $(>300 \mathrm{~mL})$

$>3$-Level Procedures

Abbreviations: ASA, American Society of Anesthesiologists; ASC, ambulatory surgical center; BMI, body mass index; CHF, congestive heart failure; EBL, estimated blood loss; NYHA, New York Heart Association.

${ }^{a}$ Interpret in the context of other comorbidities; obesity alone has been observed to have no impact on cervical surgery outcome $\mathrm{e}^{16,17,18}$; others have observed BMI $>42$ to contribute to increased $\operatorname{costs}^{19}$; low BMI $(\leq 24)$ has been found to increase chances of hematoma. $^{8}$

lower BMI $(\leq 24)[R R]=2.11, \mathrm{ASA} \geq 3[\mathrm{RR}]=1.67$, and male sex $[R R]=1.67$. Similarly, patients with BMIs greater than $40 \mathrm{~kg} / \mathrm{m}^{2}$ undergoing cervical spine procedures have an increased risk of experiencing postoperative pulmonary embolism or deep vein thrombosis (odds ratio $=3.34$ ) ${ }^{20}$ Adamson et $\mathrm{al}^{10}$ performed a retrospective review of patients undergoing ACDF both in the outpatient ambulatory center and inpatient setting. They reported ${ }^{10} \mathrm{a}$ mean age of 49.5 years, a female majority $(51.6 \%)$, and the majority having ASA $\leq 2$. They found that patients undergoing 3 or more levels had a higher risk of experiencing cervical hematomas and dysphagia, ${ }^{10}$ a finding that has been supported in other research. ${ }^{16}$ Efforts made to minimize operative time $(<5$ hours $)$ and blood loss $(<300 \mathrm{~mL})$ may successfully reduce the risk of cervical hematoma and dysphagia, which can result in prolonged intubation and reintubation when present. ${ }^{22}$

The patient cohort in the present study was consistent with those of similar research in that patients were generally non-obese (mean BMI of $28.6 \mathrm{~kg} / \mathrm{m}^{2}$ ), healthy (majority of patients with ASA score $\leq 2$ ), mostly women, aged less than 50 years, and undergoing 1- or 2-level procedures. Considering the findings of the present study and the recent pertinent literature, we have proposed the following exclusion criteria for cervical spine surgery in the ambulatory setting: age greater than 50 years; BMI less than $24 \mathrm{~kg} / \mathrm{m}^{2}$ or greater than $40 \mathrm{~kg} / \mathrm{m}^{2}$; INR greater than 1.2, ASA score greater than or equal to 2 , and preoperative comorbid conditions such as asthma, New York Heart Association grade $\geq 3$ congestive heart failure, myocardial infarction within 6 months, angina pectoris, or nonadherent obstructive sleep apnea (Table 7). ${ }^{9}$

\section{ASC Considerations}

Performing cervical surgery within the ASC raises a number of unique considerations and challenges. Surgeons must consider their patient population, the extent of surgery performed in regards to the degree of preoperative pathology and number of levels operated on, whether the facility has contracts with specific medical device companies, and personal experience of the surgeon. There is also concern regarding internal and financial bias, because several studies have suggested that physician ownership of ASCs may influence practice patterns and surgical efficiencies. $^{23,24}$ Despite potential shortcomings and conflicting interests, inherent attributes of the ASC appear to contribute to improved efficiency. Dedicated staff and operating rooms have contributed to decreased operative duration, estimated blood loss, and increased efficiency. ${ }^{25-27}$ Furthermore, single-specialty ASCs have been associated with even lower rates of surgical site infection than multispecialty facilities. ${ }^{28}$

\section{Number of Levels Involved in Operation}

When assessing cervical spine surgery, both ACDF and CDR procedures are generally welltolerated with positive outcomes. For either procedure, a critical presurgical consideration is the number of levels involved in the operation. Single-level ACDF, for example, is one of the most common spine surgeries performed, with generally good outcomes. Given its generally short operative duration, manageable postoperative pain, and a relatively lower requirement for postoperative care, it has been successfully adopted in the outpatient setting. ${ }^{29}$ However, considerable concern remains about performing multilevel surgery.

The potential for a postoperative retropharyngeal hematoma is one of the most feared complications associated with ACDF procedures, particularly those involving multiple operative levels. Few studies have investigated the association of multilevel ACDF and retropharyngeal hematoma in the outpatient setting. Although hematomas can occur days after surgery, studies have suggested that these clinically severe hematomas tend to be detected 
within 4 to 6 hours postoperatively. ${ }^{30,31}$ Whereas research suggests that a 6-hour observational period is the maximum time required after ACDF procedures, the same studies either do not specify the number of levels operated upon or are based on single-level procedures. Unfortunately, studies examining the relationship between multilevel ACDF and postoperative complications such as retropharyngeal hematoma tend to be based on large institutional datasets, ${ }^{32-34}$ and 3-level case investigations are especially limited. ${ }^{29}$ On the basis of our findings, when patients are selected and counseled appropriately, a multilevel ACDF can be safely performed in the ASC setting.

\section{Postoperative Consideration}

\section{Pain Management}

The enhanced MMA protocol used within the ASC setting is integral to delivering effective analgesia and adequately managing pain in the perioperative setting. At the heart of a successful MMA protocol is the principle that several analgesics used in a timely combination can result in a synergistic effect. This approach is able to overcome several difficulties that more conventional analgesic techniques still face. First, the use of several medications allows for the synergistic targeting of numerous unique pain pathways. In addition, because MMA is underpinned by more than 1 medication, a lower dose of each medication can be used, thereby minimizing side-effect profiles. Not only does this allow for less usage of potentially habit-forming narcotics, but it also facilitates reduced dosages of medications that could impair patient recovery. An additional advantage unique to spine and orthopedic surgery is the ability to reduce the use of nonsteroidal antiinflammatory medications due to concerns with impaired arthrodesis. ${ }^{35}$

\section{Postoperative Nausea and Vomiting}

A potential obstacle to discharge experienced by 4 of the patients in this study was the development of postoperative nausea and vomiting (PONV). ${ }^{36} \mathrm{~A}$ significant cause of PONV is the administration of opioid medications, which may be prevented through generous application of local anesthetic intraoperatively and postoperative nonnarcotic medications. $^{36,37}$ Management of PONV includes preoperative administration of antiemetics (ondansetron or metoclopramide) and adequate hydration. $^{9}$

\section{Postoperative Serotonin Syndrome}

A single patient experienced postoperative serotonin syndrome, which was a result of preoperative illicit drug use. The classical triad of symptoms includes neuromuscular abnormalities, altered mental status, and autonomic hyperactivity. ${ }^{38}$ Intraoperatively and postoperatively it may be challenging to recognize these symptoms. ${ }^{39} \mathrm{~A}$ key is identifying medications that can contribute to these symptoms. These include common antidepressants (citalopram, fluoxetine, venlafaxine, trazodone), abused opioids (fentanyl, methadone), illicit drugs (MDMA, LSD), and many others. ${ }^{38,40}$ After clinically diagnosing serotonin syndrome, management includes removing the offending medication, supportive therapy, and administration of a $5-\mathrm{HT}_{2 \mathrm{~A}}$ antagonist such as cyproheptadine. $^{38}$

\section{CONCLUSIONS}

This case series is the one of the largest to date of patients undergoing anterior cervical spine surgery within the ASC with no planned 23-hour observation period. With appropriate patient selection, surgical technique, and a MMA protocol, we were able to effectively perform both ACDF and CDR in the ASC setting. In total, 175 of 178 of the assessed patients were discharged from the surgical center on the day of surgery, and pain was adequately controlled for all patients. Disability scores universally improved in response to surgery, and the few complications were rapidly identified and appropriately treated. For an appropriately chosen patient population, it appears as though outpatient cervical spine surgery with an MMA protocol is a safe and effective treatment option.

\section{REFERENCES}

1. Kurd MF, Kreitz T, Schroeder G, Vaccaro AR. The role of multimodal analgesia in spine surgery. $J$ Am Acad Orthop Surg. 2017;25(4):260-268. doi:10.5435/jaaos-d-16-00049

2. Lemos P, Pinto A, Morais G, et al. Patient satisfaction following day surgery. J Clin Anesth. 2009;21(3):200-205. doi:10.1016/j.jclinane.2008.08.016

3. Mundell BF, Gates MJ, Kerezoudis P, et al. Does patient selection account for the perceived cost savings in outpatient spine surgery? A meta-analysis of current evidence and analysis from an administrative database. $J$ Neurosurg Spine. 2018;29(6):687-695. doi:10.3171/2018.4.spine1864

4. Martin CT, Pugely AJ, Gao Y, Mendoza-Lattes S. Thirtyday morbidity after single-level anterior cervical discectomy and fusion. J Bone Joint Surg. 2014;96(15):1288-1294. doi:10.2106/ jbjs.m.00767 
5. McClelland S, Oren JH, Protopsaltis TS, Passias PG. Outpatient anterior cervical discectomy and fusion: a metaanalysis. J Clin Neurosci. 2016;34:166-168. doi:10.1016/j.jocn. 2016.06.012

6. Pugely AJ, Martin CT, Gao Y, Mendoza-Lattes SA. Outpatient surgery reduces short-term complications in lumbar discectomy. Spine. 2013;38(3):264-271. doi:10.1097/brs. 0b013e3182697b57

7. Prabhakar A, Cefalu JN, Rowe JS, Kaye AD, Urman RD. Techniques to optimize multimodal analgesia in ambulatory surgery. Curr Pain Headache Rep. 2017;21(5). doi:10.1007/ s11916-017-0622-Z

8. Bovonratwet $\mathrm{P}, \mathrm{Fu} \mathrm{MC}$, Tyagi $\mathrm{V}$, et al. Incidence, risk factors, and clinical implications of postoperative hematoma requiring reoperation following anterior cervical discectomy and fusion. Spine. 2019;44(8):543-549. doi:10.1097/brs. 0000000000002885

9. Mohandas A, Summa C, Worthington WB, et al. Best practices for outpatient anterior cervical surgery. Spine. 2017;42(11). doi:10.1097/brs.0000000000001925

10. Adamson T, Godil SS, Mehrlich M, Mendenhall S, Asher AL, McGirt MJ. Anterior cervical discectomy and fusion in the outpatient ambulatory surgery setting compared with the inpatient hospital setting: analysis of 1000 consecutive cases. $J$ Neurosurg Spine. 2016;24(6):878-884. doi:10.3171/2015.8. spine14284

11. Lim S, Carabini LM, Kim RB, Khanna R, Dahdaleh NS, Smith ZA. Evaluation of American Society of Anesthesiologists classification as 30-day morbidity predictor after singlelevel elective anterior cervical discectomy and fusion. Spine $J$. 2017;17(3):313-320. doi:10.1016/j.spinee.2016.09.018

12. Chin KR, Pencle FJ, Coombs AV, Packer CF, Hothem EA, Seale JA. Eligibility of outpatient spine surgery candidates in a single private practice. Clin Spine Surg. 2017;30(10). doi:10. 1097/bsd.0000000000000374

13. Delsole EM, Makanji HS, Kurd MF. Current trends in ambulatory spine surgery: a systematic review. J Spine Surg. 2019;5(S2). doi:10.21037/jss.2019.04.12

14. Mathis MR, Naughton NN, Shanks AM, et al. Patient selection for day case-eligible surgery. Anesthesiology. 2013;119(6):1310-1321. doi:10.1097/aln.0000000000000005

15. Sebastian AS, Currier BL, Clarke MJ, Larson D, Huddleston PM 3rd, Nassr A: Thromboembolic Disease after Cervical Spine Surgery: A Review of 5,405 Surgical Procedures and Matched Cohort Analysis. Global Spine J. 2016; 6:465-471

16. Chotai S, Sielatycki JA, Parker SL, Sivaganesan A, Kay HL, Stonko DP, et al: Effect of obesity on cost per qualityadjusted life years gained following anterior cervical discectomy and fusion in elective degenerative pathology. Spine J. 2016; 16:1342-1350

17. Buerba RA, Fu MC, Grauer JN: Anterior and posterior cervical fusion in patients with high body mass index are not associated with greater complications. Spine J. 2014; 14:16431653

18. van Eck CF, Regan C, Donaldson WF, Kang JD, Lee JY: The revision rate and occurrence of adjacent segment disease after anterior cervical discectomy and fusion: a study of 672 consecutive patients. Spine. 2014; 39:2143-2147

19. Fabricant PD, Seeley MA, Rozell JC, Fieldston E, Flynn JM, Wells LM, et al: Cost Savings From Utilization of an
Ambulatory Surgery Center for Orthopaedic Day Surgery. $J$ Am Acad Orthop Surg. 2016; 24:865-871

20. Kalanithi PA, Arrigo R, Boakye M. Morbid obesity increases cost and complication rates in spinal arthrodesis. Spine. 2012;37(11):982-988. doi:10.1097/brs.0b013e31823bbeef

21. Li Z, Li G, Chen C, et al. Risk factors for dysphagia after anterior cervical spine surgery. Orthopedics. 2017;41(1). doi:10. 3928/01477447-20171213-04

22. Kim M, Rhim SC, Roh SW, Jeon SR. Analysis of the risk factors associated with prolonged intubation or reintubation after anterior cervical spine surgery. J Korean Med Sci. 2018;33(17). doi:10.3346/jkms.2018.33.e77

23. Mitchell JM. Do financial incentives linked to ownership of specialty hospitals affect physicians? Practice patterns? Med Care. 2008;46(7):732-737. doi:10.1097/mlr.0b013e31817892a7

24. Mitchell JM. Effect of physician ownership of specialty hospitals and ambulatory surgery centers on frequency of use of outpatient orthopedic surgery. Arch Surg. 2010;145(8):732. doi:10.1001/archsurg.2010.149

25. Kadhim M, Gans I, Baldwin K, Flynn J, Ganley T. Do surgical times and efficiency differ between inpatient and ambulatory surgery centers that are both hospital owned? $J$ Pediatr Orthop. 2016;36(4):423-428. doi:10.1097/bpo. 0000000000000454

26. Small TJ, Gad BV, Klika AK, Mounir-Soliman LS, Gerritsen RL, Barsoum WK. Dedicated orthopedic operating room unit improves operating room efficiency. J Arthroplasty. 2013;28(7). doi:10.1016/j.arth.2013.01.033

27. Avery DM, Matullo KS. The efficiency of a dedicated staff on operating room turnover time in hand surgery. $J$ Hand Surg. 2014;39(1):108-110. doi:10.1016/j.jhsa.2013.09.039

28. Mitchell P, Gottschalk M, Butts G, Xerogeanes J. Surgical site infection: a comparison of multispecialty and single specialty outpatient facilities. J Orthop. 2013;10(3):111114. doi:10.1016/j.jor.2013.07.005

29. Vaishnav A, Hill P, McAnany S, et al. Comparison of multilevel anterior cervical discectomy and fusion performed in an inpatient versus outpatient setting. Global Spine J. 2019;9(8):834-842. doi:10.1177/2192568219834894

30. Lied B, Sundseth J, Helseth E. Immediate (0-6 h), early $(6-72 \mathrm{~h})$ and late $(>72 \mathrm{~h})$ complications after anterior cervical discectomy with fusion for cervical disc degeneration; discharge six hours after operation is feasible. Acta Neurochir (Wein). 2007;150(2):111-118. doi:10.1007/s00701-007-1472-y

31. Garringer SM, Sasso RC. Safety of anterior cervical discectomy and fusion performed as outpatient surgery. $J$ Spinal Disord Tech. 2010;23(7):439-443. doi:10.1097/bsd. 0b013e3181bd0419

32. Fu MC, Gruskay JA, Samuel AM, et al. Outpatient anterior cervical discectomy and fusion is associated with fewer short-term complications in one- and two-level cases. Spine. 2017;42(14):1044-1049. doi:10.1097/brs.0000000000001988

33. McGirt MJ, Godil SS, Asher AL, Parker SL, Devin CJ. Quality analysis of anterior cervical discectomy and fusion in the outpatient versus inpatient setting: analysis of 7288 patients from the NSQIP database. Neurosurg Focus. 2015;39(6). doi:10. 3171/2015.9.focus 15335

34. McClelland S, Passias PG, Errico TJ, Bess RS, Protopsaltis TS. Outpatient anterior cervical discectomy and fusion: an analysis of readmissions from the New Jersey State 
Ambulatory Services Database. Int J Spine Surg. 2017;11(1):3. doi: $10.14444 / 4003$

35. Thaller J, Walker M, Kline AJ, Anderson DG. The effect of nonsteroidal anti-inflammatory agents on spinal fusion. Orthopedics. 2005;28(3):299-303. doi:10.3928/0147-744720050301-15

36. Buvanendran A, Thillainathan V. Preoperative and postoperative anesthetic and analgesic techniques for minimally invasive surgery of the spine. Spine. 2010;35(suppl). doi:10. 1097/brs.0b013e31820240f8

37. Nagarekha D, Shaikh S, Hegade G, Marutheesh M. Postoperative nausea and vomiting: a simple yet complex problem. Anesth Essays Res. 2016;10(3):388. doi:10.4103/02591162.179310

38. Boyer EW, Shannon M. The serotonin syndrome. $N$ Engl $J$ Med. 2005;352(11):1112-1120. doi:10.1056/nejmra041867

39. Werneke U, Jamshidi F, Taylor DM, Ott M. Conundrums in neurology: diagnosing serotonin syndrome - a metaanalysis of cases. BMC Neurol. 2016;16(1). doi:10.1186/s12883016-0616-1

40. Pedavally S, Fugate JE, Rabinstein AA. Serotonin syndrome in the intensive care unit: clinical presentations and precipitating medications. Neurocrit Care. 2013;21(1):108-113. doi:10.1007/s12028-013-9914-2

Disclosures and COI: The authors received no funding for this study and report no conflicts of interest.

Corresponding Author: Kern Singh, MD, Professor, Department of Orthopaedic Surgery, Rush University Medical Center, 1611 West Harrison St, Suite 300, Chicago, IL 60612. Phone: (312) 432-2373; Fax: (708) 409-5179; Email: kern.singh@ rushortho.com.

Published 16 April 2021

This manuscript is generously published free of charge by ISASS, the International Society for the Advancement of Spine Surgery. Copyright (c) 2021 ISASS. To see more or order reprints or permissions, see http://ijssurgery.com. 